\title{
La experiencia de la incorporación de estudiantes a un proyecto de extensión universitaria. El caso del Proyecto Formación de Formadores en Robótica en colegios en áreas vulnerables de Costa Rica
}

\author{
The Experience of Incorporating Students Into a \\ University Extension Project. The Case of the Training \\ of Trainers in Robotics Project in Schools Located in \\ Vulnerable Areas of Costa Rica
}

Irene Hernández Ruiz

Universidad Nacional

Heredia, Costa Rica

irene.hernandez.ruiz@una.cr

Richard Arce Vargas

Universidad Nacional

Heredia, Costa Rica richard1996.arce@gmail.com

Pedro Fonseca Solano

Universidad Nacional

Heredia, Costa Rica

pedro.fonseca.solano@una.cr

Juan Pablo Vargas González

Calidad de estudiante

Universidad Nacional

Heredia, Costa Rica

juanpi1173@gmail.com

Hilary Granados Álvarez

Universidad Nacional

Heredia, Costa Rica

hilary.granalva13@gmail.com

Recibido 31/05/19 Aceptado:30/08/2019 
Resumen. El presente trabajo da a conocer la experiencia de los estudiantes que colaboran en el Proyecto Formación de Formadores en Robótica en colegios en áreas vulnerables de Costa Rica, un proyecto de extensión universitaria de la Universidad Nacional que desde el 2015 se encuentra trabajando en el área de la robótica educativa. Además, se presenta el impacto que este proyecto ha tenido en su formación como futuros profesionales en el área de la ingeniería en sistemas de información.

Palabras clave: Lego Digital Designer, robótica, Lego Ev3, mentoría.

Abstract. This work describes the experience of students who collaborate in the Training of Trainers in Robotics project in schools located in vulnerable areas of Costa Rica. This is a university extension project of the National University, which has been implemented in the field of educational robotics since 2015. Besides, this paper describes the impact this project has had on the participants' training as future professionals in the area of information systems engineering.

Keywords: Lego Digital Designer, robotics, Lego Ev3, mentoring.

\section{Introducción}

El Proyecto Formación de Formadores en Robótica en colegios en áreas vulnerables de Costa Rica es un proyecto de extensión universitaria de la Escuela de Informática (ESCINF) de la Universidad Nacional, el cual tiene como objetivo la formación de docentes en el área de la robótica educativa, para que trabajen en colegios en áreas vulnerables y que, a su vez, repliquen la experiencia y sus conocimientos con sus estudiantes (Fonseca y Hernández, 2017). Este es un proyecto que se desarrolla de forma conjunta con el Instituto Costarricense sobre Drogas y el Ministerio de Educación Pública de Costa Rica.

Es un proyecto innovador en su campo y hasta el momento ha capacitado a 36 docentes en el área de la tecnología y sobre la capacidad que tiene la robótica para enseñar a los estudiantes a programar y que conozcan los beneficios que les puede brindar esta disciplina. Además, hasta el momento, estos 36 docentes han logrado capacitar a más de mil estudiantes de secundaria.

También, se realizó en el 2017 una Olimpiada Nacional en la que los estudiantes beneficiarios del proyecto pusieron en práctica el conocimiento adquirido a través de los talleres de robótica que sus docentes brindaron. Se logró la participación de 202 estudiantes, de los cuales 56 eran mujeres, lo que representa un $28 \%$ y 146 hombres, que corresponde a un $72 \%$. 
URL: http://www.revistas.una.ac.cr/index.php/dialogo/index

CORREO ELECTRÓNICO: universidadendialogo@una.cr

DOI: http://doi.org/10.15359/udre.9-2.8

Para el proyecto siempre ha sido importante promover la participación de las mujeres en el área de la tecnología, así como también para otras instituciones del país; por ejemplo, en el año del 2011, la vicepresidenta de la Cámara Costarricense de Tecnologías de Información y Comunicación (CAMTIC) recalcó la importancia de que cada vez más mujeres descubran que las carreras ligadas a las ingenierías son opciones viables para ellas (CAMTIC, 2011).

La ESCINF actualmente es una de las escuelas que posee la mayor cantidad de estudiantes de toda la Universidad Nacional, por lo cual la incorporación de estudiantes universitarios a diferentes proyectos internos es una oportunidad única para los mismos, ya que pueden aplicar, entre otras cosas, los conocimientos que han aprendido en su formación en la carrera y el desarrollo de habilidades blandas que les serán de utilidad para su futuro como profesionales. Para el desarrollo de los talleres se volvió indispensable la colaboración de estudiantes propios de la escuela para que apoyaran durante los mismos. Y también para atender junto con los académicos las solicitudes de giras educativas por parte de docentes de secundaria para traer a sus estudiantes a conocer la institución y para recibir un taller de robótica.

Los estudiantes asistentes han existido durante muchos años, por eso es importante tomar en cuenta toda su experiencia, ya que muchas veces los estudiantes asistentes tienen un contacto más amistoso con otros estudiantes que con los profesores, debido a que el intercambio de ideas entre estudiantes se puede realizar más cómodamente y en un ambiente relajado (Dickson, 2011). En este proyecto sucede igual, al trabajar con varios estudiantes asistentes, se ha formado un grupo en el cual hay confianza entre los compañeros, gracias a que, al haber diferentes estudiantes, estos se sentían menos presionados.

Además, no solo tenemos que enfocarnos en el aprendizaje de los estudiantes, sino también en el aprendizaje que los profesores adquieren, con reuniones y con el desarrollo de trabajos grupales para compartir conocimientos entre el profesor y el estudiante, lo cual es beneficioso en términos de mejorar en el campo personal y profesional para ambas partes (Patitsas \& Wolfman, 2013).

Cabe mencionar que los estudiantes asistentes han trabajado desempeñándose y adaptándose a nuevas tareas, además de trabajar en proyectos que para ellos pueden considerarse nuevos. Por otra parte, la motivación que provoca que ellos trabajen de manera correcta siempre va de la mano con el ambiente de trabajo que los rodea, por lo cual las reuniones que se realizan mes a mes construyeron un ambiente cómodo, ayudando a que ellos tuvieran más satisfacción en el trabajo que realizaron (Patitsas, 2013). 
Revista Universidad en Diálogo • Vol. 9, N. ${ }^{\circ}$ 2, Julio-Diciembre, 2019 • 161-173

ISSN 2215-2849 • EISSN: 2215-4752

URL: http://www.revistas.una.ac.cr/index.php/dialogo/index CORREO ELECTRÓNICO: universidadendialogo@una.cr DOI: http://doi.org/10.15359/udre.9-2.8

De acuerdo con Puga y Martínez (2008), las competencias más demandadas por el mercado laboral actual se vinculan más con capacidades mentales y/o personales -llamadas habilidades blandas- que con destrezas mecánicas o prácticas.

Para Gil et al. (2013), aprender a desarrollar las habilidades sociales resulta fundamental en la formación del estudiantado, en la búsqueda de mantener buenas relaciones con personas en los diferentes contextos de su vida.

Por otra parte, actualmente existen metodologías como el aprendizaje activo, el cual es una aproximación metodológica centrada en el estudiantado, con la premisa de que el conocimiento se construye a partir de la interacción con los demás individuos, apoyándose en la reflexión y las vivencias situadas en un contexto determinado (Howell, citado por Koo, 1999).

\section{Desarrollo de la experiencia con estudiantes}

Este proyecto comenzó a mediados del 2015 como una respuesta novedosa para ofrecer a los docentes de los colegios en zonas vulnerables capacitación en el área de robótica, para que pudieran ofrecer talleres a sus estudiantes. La incorporación de estudiantes fue de gran ayuda para el proyecto, pero lo más importante fue la ayuda para ellos mismos, al momento de ingresar al proyecto en modalidad de estudiante asistente. Los estudiantes tuvieron que adaptarse rápidamente a la metodología de trabajo del proyecto, es decir, seguir el ritmo en las diferentes actividades en un periodo de tiempo relativamente corto. Al ser un proyecto enfocado en robótica - un área en la Escuela de Informática que solo se ofrece como curso optativo-, los estudiantes tuvieron que recibir pequeñas capacitaciones para lograr actualizarse y colaborar con los talleres, por lo cual no entender sobre un tema no era una opción, los estudiantes asistentes aprendieron sobre la práctica.

Los estudiantes respondieron de manera positiva durante los talleres y lograron dominar los conceptos básicos en un periodo de un mes. Los talleres se realizaron semanalmente, mientras los estudiantes siguieron adaptándose, creando nuevas ideas y colaborando.

Con el paso de los meses, se empezó a crear un equipo de trabajo, en donde cada estudiante tenía sus funciones establecidas y las realizaba correctamente. El gran crecimiento de los estudiantes en áreas como el trabajo en equipo, trabajar bajo presión, ser amigable, capacidad de aprendizaje, la fluidez para comunicarse, la comunicación asertiva y la dedicación hacia el trabajo son habilidades que mejoraron considerablemente. 
URL: http://www.revistas.una.ac.cr/index.php/dialogo/index

CORREO ELECTRÓNICO: universidadendialogo@una.cr

DOI: http://doi.org/10.15359/udre.9-2.8

Los estudiantes no solo mejoraron habilidades blandas, también mejoraron sus habilidades técnicas en el área de la informática, por ejemplo, la comprensión de conceptos básicos de programación, desarrollo de programas para los robots, la utilización de nuevos instrumentos como la herramienta de Lego son características que dan un valor agregado al conocimiento adquirido por los estudiantes.

En los proyectos de extensión muchas veces pasan desapercibidos, pero son una gran oportunidad para los estudiantes de aprender, es un aprendizaje en habilidades blandas, es decir, cómo tratar a personas, cómo trabajar en equipo, cómo comunicarse de la mejor manera. Es un conjunto de habilidades que se empiezan a formar de manera temprana, para que el día en que los estudiantes trabajen estén preparados para el ambiente laboral.

En la figura 1, se muestra una imagen de la explicación de un estudiante asistente durante uno de los talleres:

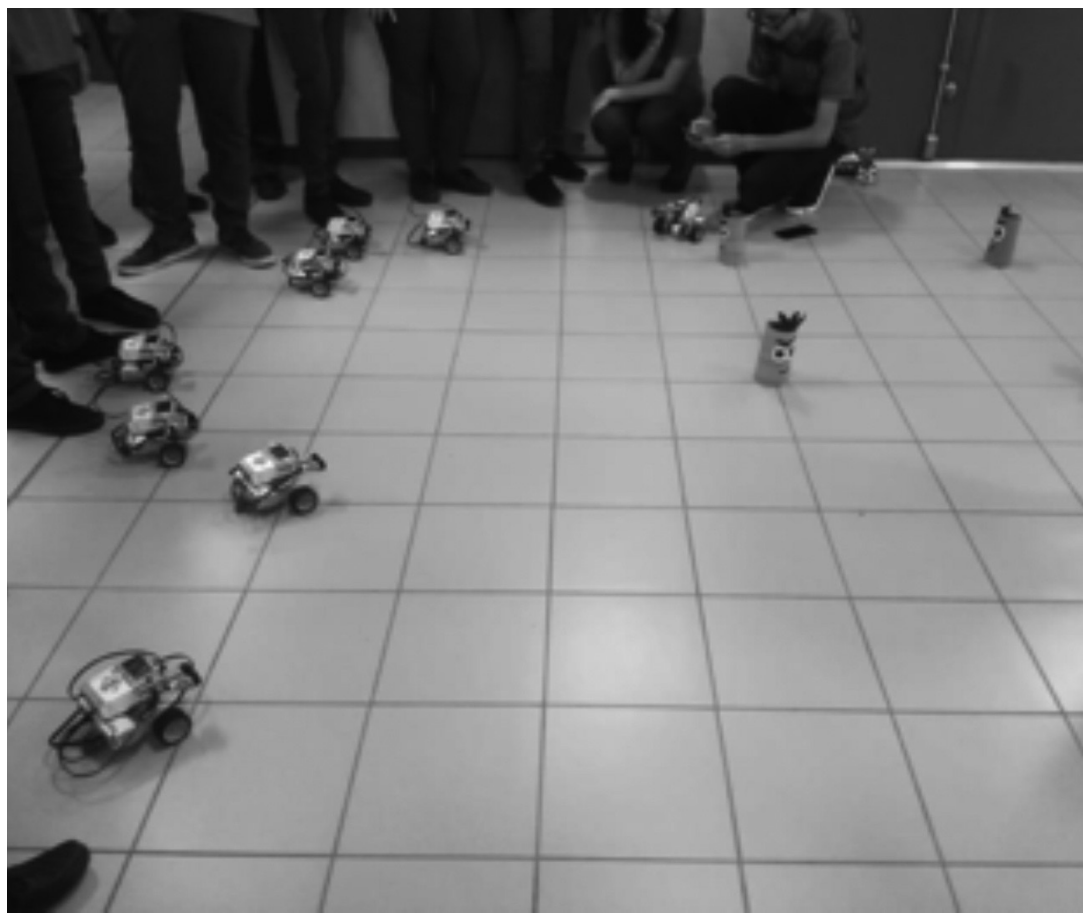

Figura 1. Explicación del uso de los robots con los teléfonos.

Fuente: elaboración propia. 
En la figura 3 se muestra a uno de los estudiantes asistentes explicándole a una estudiante cómo se puede hacer uso del robot con el teléfono. Esto para participar en una competencia con los teléfonos para esquivar los vasos de color morado. Además, se visualizan varios robots activos, los cuales ya se encuentran listos para la competencia.

Actualmente, hay seis estudiantes voluntarios, de los cuales dos son de cuarto año, uno de tercer año, dos de segundo año y uno de primer año. Con una edad promedio de veintidós años; cuatro son hombres y dos son mujeres.

\section{Entorno de trabajo}

Para la realización de los talleres de capacitación a los docentes de los colegios, los estudiantes asistentes colaboraron en cada uno de los talleres. Para los mismos, se utilizaron los kits de Lego Mindstorm EV3 Education, se utilizaron diferentes modelos de robots haciendo uso de equipo LEGO EV $3^{1}$. Este kit de piezas LEGO permite la construcción de diferentes modelos de robots. Además, ofrece un lenguaje gráfico para programar modelos de robots construidos. Esto mediante un conjunto de recursos gráficos (bloques) que permiten incorporar los conceptos y estructuras de programación de una manera muy intuitiva.

Por otra parte, se utilizaron otras herramientas de Lego, como Lego Digital Designer para la elaboración de guías de cómo construir los robots. En la figura 2, se presenta una imagen de la construcción que se puede realizar en este entorno:



Figura 2. Estructura de programación por bloques que presenta la herramienta de Lego. Fuente: elaboración propia. 
URL: http://www.revistas.una.ac.cr/index.php/dialogo/index

CORREO ELECTRÓNICO: universidadendialogo@una.cr

DOI: http://doi.org/10.15359/udre.9-2.8

Además, también los estudiantes asistentes aprendieron a utilizar el software Lego Mindstorm Home Edition para la programación de los robots.



Figura 3. Estructura de programación por bloques que presenta la herramienta de Lego. Fuente: elaboración propia.

\section{Metodología}

Con el fin de conocer la perspectiva de los estudiantes sobre este proyecto, se procedió a elaborar un cuestionario en Google Forms para que los colaboradores asistentes lograran expresar sus comentarios. Para lo cual se contó con la respuesta de los seis estudiantes que han colaborado con el proyecto durante los años 2015, 2016 y 2017. Para representar el cuestionario se elaboró la tabla 1, en el lado izquierdo se encuentra el dato solicitado y en el lado derecho las opciones, si el campo está vacío significa que la respuesta era libre.

Tabla 1

Cuestionario aplicado a los estudiantes

\begin{tabular}{|l|l|}
\hline Nombre & \\
\hline Sexo & $\begin{array}{l}\text { Masculino } \\
\text { Femenino }\end{array}$ \\
\hline Tipo de horas & $\begin{array}{l}\bullet \text { Horas asistente } \\
\bullet \text { Horas colaboración }\end{array}$ \\
\hline $\begin{array}{l}\text { ¿Desde cuándo colabora con el } \\
\text { proyecto? }\end{array}$ & \\
\hline ¿Qué le gusta más del proyecto? & \\
\hline $\begin{array}{l}\text { ¿Considera que es un ambiente } \\
\text { agradable para compartir con sus } \\
\text { compañeros? }\end{array}$ & \\
\hline $\begin{array}{l}\text { ¿Cuántas horas colaboró en el } \\
\text { proyecto? }\end{array}$ & \\
\hline $\begin{array}{l}\text { ¿Lleva el bloque universitario } \\
\text { completo? }\end{array}$ & \\
\hline
\end{tabular}

continúa... 
Revista Universidad en Diálogo • Vol. 9, N. ${ }^{\circ}$ 2, Julio-Diciembre, 2019 • 161-173

URL: http://www.revistas.una.ac.cr/index.php/dialogo/index CORREO ELECTRÓNICO: universidadendialogo@una.cr DOI: http://doi.org/10.15359/udre.9-2.8

\begin{tabular}{|c|c|}
\hline Año de carrera que cursa & \\
\hline $\begin{array}{l}\text { ¿Considera que los proyectos de } \\
\text { extensión son importantes? }\end{array}$ & \\
\hline ¿Cómo se enteró del proyecto? & \\
\hline $\begin{array}{l}\text { ¿Cuáles son las habilidades y } \\
\text { destrezas que le han sido de } \\
\text { utilidad en el proyecto? }\end{array}$ & \\
\hline $\begin{array}{l}\text { ¿Considera que este proyecto ha } \\
\text { sido de utilidad para su formación } \\
\text { como futuro profesional? ¿Por } \\
\text { qué? }\end{array}$ & \\
\hline $\begin{array}{l}\text { ¿Qué recomendaciones daría al } \\
\text { proyecto? }\end{array}$ & \\
\hline $\begin{array}{l}\text { ¿Considera que el uso de los } \\
\text { robots ha sido sencillo? ¿Por qué? }\end{array}$ & \\
\hline $\begin{array}{l}\text { ¿Que otro aporte daría al } \\
\text { proyecto de robótica? }\end{array}$ & \\
\hline $\begin{array}{l}\text { ¿Desde su inicio en el proyecto } \\
\text { en cuántos talleres ha colaborado } \\
\text { aproximadamente? }\end{array}$ & \\
\hline $\begin{array}{l}\text { ¿Qué actividades realizó dentro } \\
\text { del proyecto? }\end{array}$ & \\
\hline $\begin{array}{l}\text { Marque cuáles habilidades } \\
\text { blandas ha logrado desarrollar }\end{array}$ & $\begin{array}{l}\text { - Me es más fácil comunicarme con otros. } \\
\text { - Puedo expresar mejor mis ideas. } \\
\text { - Me siento más a gusto con las personas que estudian } \\
\text { lo mismo que yo. } \\
\text { - Considero que ahora comprendo mejor los conceptos } \\
\text { de programación. } \\
\text { - Trabajo en equipo. } \\
\text { - Ser consciente de que mi colaboración en un } \\
\text { trabajo de equipo es determinante para lograr el } \\
\text { éxito o no. } \\
\text { - Me ha permito tenerme más confianza. } \\
\text { - Me permite tener un sentido de permanencia. } \\
\text { - Me brinda una mayor seguridad en lo que hago }\end{array}$ \\
\hline
\end{tabular}

Fuente: elaboración propia.

\section{Resultados obtenidos}

Los resultados de este proyecto han sido sumamente positivos tanto para la Universidad Nacional como para todas las personas involucradas en este proyecto, en especial los jóvenes a los que los profesores capacitados por la Universidad les impartieron el taller. 
A continuación, se presentan los principales resultados del cuestionario:

Tabla 2

Elementos que más les gustan del proyecto

\begin{tabular}{|l|}
\hline ¿Qué les gusta más del proyecto? \\
\hline «Adquirir nuevos conocimientos» \\
\hline $\begin{array}{l}\text { «Cuando se hacen los talleres es bonito ver cómo los participantes se } \\
\text { emocionan con los robots» }\end{array}$ \\
\hline «Aprender y compartir» \\
\hline «La oportunidad de aprender sobre robótica y enseñar a otras personas» \\
\hline «La enseñanza visual física y entretenida» \\
\hline
\end{tabular}

Fuente: elaboración propia.

- ¿Lleva el bloque universitario completo? $88.3 \%$ sí y el $16,7 \%$ no lleva el bloque completo.

- ¿Considera que los proyectos de extensión son importantes? Todos consideraron que sí. Entre los porqués se encuentra que:

«De esta manera hay más posibilidades para muchas personas de aprender de los diferentes cursos que se pueden dar».

«Claro, ayudan a los estudiantes a tener un acercamiento profesional».

- ¿Cómo se enteró del proyecto? Haciendo horas colaboración con una profesora, me recomendó una compañera, me inscribí como estudiante asistente.

- ¿Cuáles son las habilidades y destrezas que le han sido de utilidad en el proyecto?

- Trabajo en equipo

- Conocimiento en robótica, resolución de problemas

- La programación

- Creatividad, ingenio, facilidad para enseñarles a otros e iniciativa

- ¿Considera que este proyecto ha sido de utilidad para su formación como futuro profesional? ¿Por qué? 
«Por el poco tiempo que llevo en el proyecto, puedo decir que todavía necesito más conocimiento respecto al proyecto».

«Sí, ya que es una nueva experiencia y como grupo nos enfrentamos a varios retos de forma continua».

«Claro que sí, me ha dado la oportunidad de asistir a eventos, de tratar a personas de todos los rangos y también me ha dado experiencia profesional».

«He aprendido muchas cosas, probablemente me sean útiles en un futuro».

«Sí, pues ayuda a desarrollar y poner en práctica un pensamiento creativo a la hora de resolver problemas».

«Sí lo considero porque he aprendido muchas cosas nuevas gracias a este proyecto».

- ¿Considera que el uso de los robots ha sido sencillo? ¿Por qué?

«No me parece algo del otro mundo, sí es sencillo; ya que soy estudiante de Ingeniería en Sistemas, pero en el caso de que sea para otras personas sin el conocimiento necesario, puede ser diferente. Pero es accesible totalmente de aprender».

«Sí, ya que la utilización del equipo Lego es relativamente fácil de entender».

«Al principio fue difícil acostumbrarse, pero luego fue más sencillo». «Es divertido y eso hace que no se le presente tanta atención a la dificultad».

«Sí, porque se tienen bases muy bien explicadas y un constante apoyo».

«Sí, es sencillo porque uno le toma el gusto a hacer y usar los robots».

- ¿Cuáles habilidades blandas ha logrado desarrollar?

«Trabajo en equipo, me siento más a gusto con las personas que estudian lo mismo que yo».

«Puedo expresar mejor mis ideas».

«Consideroqueahora comprendomejor los conceptos de programación». 


\section{Conclusiones}

Luego de haber realizado la documentación anterior y analizar las experiencias vividas y conocimientos obtenidos durante el trayecto del aprendizaje, algunas de las conclusiones más relevantes son:

- La incorporación de nuevos estudiantes al ambiente académico es una gran oportunidad para los jóvenes estudiantes para que se desenvuelvan en un ambiente profesional, además son preparados de mejor manera para el mercado laboral.

- Los jóvenes participantes del proyecto están acercándose al área de la robótica, dejando de lado las malas influencias; este tipo de modelos un poco más avanzados son una distracción sana para que los estudiantes puedan desarrollar habilidades como la lógica y la creatividad.

- Algunas veces estas personas tienen ideas nuevas que a su vez son buenas para el desarrollo de nuevos modelos. Al ser jóvenes tienen una perspectiva diferente que puede ser innovadora en muchos aspectos. Esto mismo da paso a que el estudiante tenga la oportunidad de relacionarse con personas profesionales de las cuales puede adquirir gran cantidad de conocimiento, también adquiere un grado de experiencia que le será útil en su carrera y a lo largo de su vida, y le ayuda a prepararse para afrontar nuevos proyectos en el futuro. De esa forma, el estudiante puede darse a conocer junto con el proyecto y si ha tenido un buen desarrollo, puede optar por grandes oportunidades.

- Incluir jóvenes estudiantes al proyecto les ha ayudado para acostumbrarse a tratar con profesionales, provocando que tuvieran una curva de aprendizaje basada en la experiencia laboral de los diferentes talleres que se realizaron.

- Es importante para la Escuela de Informática que sus estudiantes se integren a los diferentes proyectos que existen. Y gracias a la extensión universitaria los estudiantes asistentes le retribuyen a la sociedad costarricense parte de su conocimiento y motivan a las nuevas generaciones sobre la importancia del estudio. 


\section{Recomendaciones}

- Realizar talleres relacionados con el ensamblaje de piezas de manera avanzada, para así animar a los jóvenes a que intenten nuevas combinaciones.

- Promover el intercambio de conocimientos entre los profesores y los estudiantes para la realización de modelos como el que estamos presentando.

- Construir en los talleres modelos más avanzados para motivar a los jóvenes a que con solo modificar un par de piezas pueden llegar la curiosidad y las ganas de crear.

- Incorporar más estudiantes asistentes a proyectos académicos de la universidad, ya que la experiencia que brindan estos es única, la posibilidad de obtener conocimiento que solo se consigue con vivencias es algo que no tiene precio, no solo ayuda en el campo profesional a los estudiantes, sino que también es una motivación para seguir en la carrera y seguir en la universidad.

\section{Referencias bibliográficas}

Cámara de Tecnologías de Información y Comunicación de Costa Rica (CAMTIC) (octubre, 2011). Industria TIC ofrece mayores oportunidades para el desarrollo de las mujeres [online]. Recuperado de http:// www.camtic.org/clic/actualidad-tic/industria-tic-ofrece-mayoresoportunidades-para-el-desarrollo-de-las-mujeres/

Dickson, P. E. (2011). Using undergraduate teaching assistants in a small college environment. Proceedings of the 42nd ACM Technical Symposium on Computer Science Education - SIGCSE '11, 75. https:// doi.org/10.1145/1953163.1953187

Fonseca, P. y Hernández, I. (2017). Training of robotic trainers for schools in vulnerable areas of Costa Rica: Use of ICT to help Costa Rican youth avoid drug use. DOI: 10.1109/CLEI.2017.8226421 
Gil, P., Gutiérrez, E. C. y Madrid, P. D. (2013). Incremento de las habilidades sociales a través de la expresión corporal: la experiencia en clases de iniciación al baile. Cuadernos de Psicología del Deporte, 12(2), 83-88. Recuperado de http://revistas.um.es/cpd/article/ view/177821/149501

Koo, L. C. (1999). Learning action learning. Journal of Workplace Learning, 11(3), 89-94. doi: https://doi.org/10.1108/13665629910264244

Patitsas, E. A. \& Wolfman, S. A. (2013). A case study of the development of CS teaching assistants and their experiences with team teaching. Proceedings of the 13th Koli Calling International Conference on Computing Education Research - Koli Calling '13, 115-124. https:// doi.org/10.1145/2526968.2526981

Puga, J. y Martinez, L. (2008). Competencias directivas en escenarios globales. Estudios Gerenciales, 24(109), 87-103. doi: https://doi.org/10.1016/ S0123-5923(08)70054-8 
